\title{
HILBERT-VALUED FORMS AND BARRIERS ON WEAKLY PSEUDOCONVEX DOMAINS
}

\author{
Vincent Thilliez
}

\begin{abstract}
We introduce an alternative proof of the existence of certain $C^{k}$ barrier maps, with polynomial explosion of the derivatives, on weakly pseudoconvex domains in $\mathbb{C}^{n}$. Barriers of this sort have been constructed very recently by J. Michel and M.-C. Shaw, and have various applications. In our paper, the adaptation of Hörmander's $L^{2}$ techniques to suitable vector-valued functions allows us to give a very simple approach of the problem and to improve some aspects of the result of Michel and Shaw, regarding the explosion of the barrier and the regularity assumption on the domain.
\end{abstract}

\section{Introduction and statement of results}

Let $\Omega$ be a bounded weakly pseudoconvex domain in $\mathbb{C}^{n}$ with Lip ${ }^{1}$ boundary $\partial \Omega$ and let $\Omega^{*}$ be a bounded domain with Lip ${ }^{1}$ boundary such that $\bar{\Omega} \subset \Omega^{*}$. Put $U=\Omega^{*} \backslash \bar{\Omega}$. The aim of the present paper is to give a simple proof of the following theorem.

Main Theorem. Let $k$ be a positive integer. There exist functions $w_{1}, \ldots, w_{n}$ belonging to $C^{k}(\Omega \times \bar{U})$ and satisfying the following properties:

(1) For any $\zeta$ in $\bar{U}$ and any $j=1, \ldots, n$ the function $w_{j}(\cdot, \zeta)$ is holomorphic in $\Omega$.

(2) For any $(z, \zeta)$ in $\Omega \times \bar{U}$, one has

$$
\sum_{j=1}^{n} w_{j}(z, \zeta)\left(z_{j}-\zeta_{j}\right)=1
$$

Keywords. Barrier maps, weakly pseudoconvex domains, $C^{k}$ estimates. 1991 Mathematics subject classifications: 32A25, 35N15, 46 E99. 
(3) There exists a constant $C_{k}$, depending only on $k, \Omega$ and $\Omega^{*}$, such that the estimate

$$
\left|D_{\zeta}^{\ell} w_{j}(z, \zeta)\right| \leq C_{k} \operatorname{dist}(z, \partial \Omega)^{-\left((n+1) k+n^{2}+5 n+2\right)}
$$

holds for any $j=1, \ldots, n$, any derivation $D_{\zeta}^{\ell}$ of order $\ell \leq k$ with respect to $\zeta$, and any $(z, \zeta)$ in $\Omega \times \bar{U}$.

It is essential to remark that a quite similar result has been obtained previously by Joachim Michel and Mei-Chi Shaw [8]. However, the proof in $[\mathbf{8}]$, using the powerful machinery from [7], seems much more technical than ours. It also requires the boundary of $\Omega$ to be $C^{2}$-smooth, while we require only $\operatorname{Lip}^{1}$ regularity. Moreover, the article $[\mathbf{8}]$ yelds $C^{k}$ functions $w_{j}(z, \zeta)$ having their growth controlled by $\operatorname{dist}(z, \partial \Omega)^{-\left(a_{n} k^{2}+b_{n}\right)}$ (for suitable $\left.a_{n}, b_{n}\right)$ instead of $\operatorname{dist}(z, \partial \Omega)^{-\left(a_{n} k+b_{n}\right)}$ as in statement (3). So our version of the theorem improves the main result in $[\mathbf{8}]$ with respect to both aspects. It should be also pointed out that a so-called "barrier map" such as $\left(w_{1}, \ldots, w_{n}\right)$ has many applications, for which we refer the reader to $[\mathbf{8}],[\mathbf{9}],[\mathbf{1 0}],[\mathbf{1 1}]$. Of course, our improved barrier allows one to obtain, more or less mechanically, some improvements of these applications too (see e.g. the estimates for the solution of $\bar{\partial}$ on annuli given by Theorem 2 in [8]). The essential trick of our proof of the main theorem consists in "hiding" the parameter $\zeta \in U$ by means of Hörmander-type $L^{2}$ techniques applied to square-integrable functions taking their values in a Sobolev space $H^{m}(U)$. We organize this as follows. Section 1 gathers some properties of Hilbert-valued differential forms needed in the rest of the paper. In section 2, a classical homological device leads to a $H^{m}(U)$-valued barrier map. Then $C^{k}$ regularity and estimates for the barrier are obtained by elementary arguments, for a suitable choice of $m$.

\section{The $\bar{\partial}$-operator for Hilbert-valued forms}

1.1. Notations. Throughout this section, $\mathcal{H}$ will denote a complex separable Hilbert space, $\langle\cdot \mid \cdot\rangle_{\mathcal{H}}$ its inner product (with the convention that $\left\langle h \mid h^{\prime}\right\rangle_{\mathcal{H}}$ is linear with respect to $h^{\prime}$ ) and $\|\cdot\|_{\mathcal{H}}$ the corresponding norm. It is assumed that there exists a conjugation, in other words an antilinear isometric involution $h \in \mathcal{H} \mapsto \bar{h} \in \mathcal{H}$ (the notation $\bar{h}$ should cause no confusion). For $\left(h, h^{\prime}\right) \in \mathcal{H} \times \mathcal{H}$, put

$$
B\left(h, h^{\prime}\right)=\left\langle\bar{h} \mid h^{\prime}\right\rangle_{\mathcal{H}} .
$$

Then $B$ is clearly a continuous bilinear symmetric form on $\mathcal{H}$. Now let $q$ be an integer, $q \geq 0$. Denote by $\Lambda^{(0, q)}$ the space of $(0, q)$-forms on $\mathbb{C}^{n}$. 
Any element $\eta$ of $\Lambda^{(0, q)} \otimes \mathcal{H}$ can be written

$$
\eta=\sum_{Q} d \bar{z}^{Q} \otimes \eta_{Q}
$$

where the summation is performed for all $Q=\left(j_{1}, \ldots, j_{q}\right), 1 \leq j_{1}<$ $\cdots<j_{q} \leq n$, with the standard meaning $d \bar{z}_{j_{1}} \wedge \cdots \wedge d \bar{z}_{j_{q}}$ for $d \bar{z}^{Q}$, and with each $\eta_{Q}$ belonging to $\mathcal{H}$. Thus the inner product $\langle\cdot \mid \cdot\rangle_{\mathcal{H}}$, as well as the Hilbert norm $\|\cdot\|_{\mathcal{H}}$, extends naturally to $\Lambda^{(0, q)} \otimes \mathcal{H}$ by means of the formula

$$
\left\langle\eta \mid \eta^{\prime}\right\rangle_{\mathcal{H}}=\sum_{Q}\left\langle\eta_{Q} \mid \eta_{Q}^{\prime}\right\rangle_{\mathcal{H}}
$$

Let $\Omega$ be as in the introduction. We denote by $L^{2}(\Omega ; \mathcal{H}$, loc $)$ the space of locally square-integrable $\mathcal{H}$-valued functions in $\Omega$ (see $[\mathbf{2}]$ for the main facts about such spaces), endowed with the topology of $L^{2}$-convergence on compact subsets of $\Omega$. The corresponding space of $\mathcal{H}$-valued $(0, q)$ differential forms $L^{2}\left(\Omega ; \Lambda^{(0, q)} \otimes \mathcal{H}\right.$, loc $)$ will be written $L_{(0, q)}^{2}(\Omega ; \mathcal{H}$, loc $)$ for short. When $\mathcal{H}=\mathbb{C}$, this is the familiar space of $(0, q)$-forms with locally square-integrable coefficients used in Hörmander's $L^{2}$ theory for $\bar{\partial}$. At last, for any map $f: \Omega \longrightarrow \mathcal{H}$, any $h$ in $\mathcal{H}$ and $z$ in $\Omega$, we put

$$
f_{h}(z)=\langle h \mid f(z)\rangle_{\mathcal{H}} .
$$

Obviously, if $f$ belongs to $L^{2}(\Omega ; \mathcal{H}$, loc $)$, then $f_{h}$ belongs to $L^{2}(\Omega ; \mathbb{C}$, loc $)$.

1.2. A $\bar{\partial}$-operator. The process to define $\bar{\partial}$ on $\mathcal{H}$-valued $L^{2}$ spaces is the same as for usual $L^{2}$ spaces. Let $\mathcal{D}(\Omega ; \mathcal{H})$ be the space of $C^{\infty}$, compactly supported, $\mathcal{H}$-valued functions in $\Omega$, endowed with its standard (LF) topology. For any $f$ belonging to $L^{2}(\Omega ; \mathcal{H}$, loc $)$ and any $j=1, \ldots, n$ we define $\partial f / \partial \bar{z}_{j}$ as the continuous linear form on $\mathcal{D}(\Omega ; \mathcal{H})$ such that

$$
\left\langle\frac{\partial f}{\partial \bar{z}_{j}}, \varphi\right\rangle=-\int_{\Omega} B\left(f(z), \frac{\partial \varphi}{\partial \bar{z}_{j}}(z)\right) d V(z) \text { for all } \varphi \in \mathcal{D}(\Omega ; \mathcal{H}) .
$$

Here $d V$ denotes the standard Lebesgue measure and $\langle\cdot, \cdot\rangle$ is the duality bracket. If there exists a function $g_{j}$ in $L^{2}(\Omega ; \mathcal{H}$, loc $)$ such that the equality

$$
\int_{\Omega} B\left(g_{j}(z), \varphi(z)\right) d V(z)=-\int_{\Omega} B\left(f(z), \frac{\partial \varphi}{\partial \bar{z}_{j}}(z)\right) d V(z)
$$

holds for any $\varphi$ in $\mathcal{D}(\Omega ; \mathcal{H})$ (for instance, this is straightforward when $f$ is $C^{1}$ ), then $\partial f / \partial \bar{z}_{j}$ identifies with $g_{j}$. Hence it is possible to define

$$
\bar{\partial} f=\sum_{j=1}^{n} d \bar{z}_{j} \otimes \frac{\partial f}{\partial \bar{z}_{j}}
$$


as an element of $L_{(0,1)}^{2}(\Omega ; \mathcal{H}$, loc $)$. This definition extends in a purely algebraic way to higher degrees, giving rise to a $\bar{\partial}$-complex on $L_{(0, q)}^{2}(\Omega ; \mathcal{H}$, loc $)$ spaces. We describe now some basic rules about this complex.

1.3. Lemma. Let $f$ be a function belonging to $L^{2}(\Omega ; \mathcal{H}$, loc $)$ and such that $\bar{\partial} f$ belongs to $L_{(0,1)}^{2}(\Omega ; \mathcal{H}$, loc $)$. Then for any $h$ in $\mathcal{H}$ and any $j=1, \ldots, n$, one has, in the sense of notation (1.1.1),

$$
\frac{\partial f_{h}}{\partial \bar{z}_{j}}=\left(\frac{\partial f}{\partial \bar{z}_{j}}\right)_{h} \quad \text { in } \quad L^{2}(\Omega ; \mathbb{C}, \text { loc }) .
$$

Thus if $\bar{\partial} f$ equals zero, the function $f_{h}$ is holomorphic (in the usual sense) in $\Omega$.

Proof: For any $\psi$ in $\mathcal{D}(\Omega ; \mathbb{C})$ and any $z$ in $\Omega$, one has

$$
\begin{aligned}
f_{h}(z) \frac{\partial \psi}{\partial \bar{z}_{j}}(z)=\left\langle\overline{\frac{\partial \psi}{\partial \bar{z}_{j}}(z)} h \mid f(z)\right\rangle_{\mathcal{H}} & =\left\langle\overline{\frac{\partial \psi}{\partial \bar{z}_{j}}(z) \bar{h}} \mid f(z)\right\rangle_{\mathcal{H}} \\
& =B\left(f(z), \frac{\partial(\psi \otimes \bar{h})}{\partial \bar{z}_{j}}(z)\right),
\end{aligned}
$$

hence $\left\langle\frac{\partial f_{h}}{\partial \bar{z}_{j}}, \psi\right\rangle$ equals $\left\langle\frac{\partial f}{\partial \bar{z}_{j}},(\psi \otimes \bar{h})\right\rangle$. On the other hand, one has similarly

$$
\begin{aligned}
\left(\frac{\partial f}{\partial \bar{z}_{j}}\right)_{h}(z) \psi(z)=\left\langle\overline{\psi(z)} h \mid \frac{\partial f}{\partial \bar{z}_{j}}(z)\right\rangle_{\mathcal{H}} & =\left\langle\overline{\psi(z) \bar{h}} \mid \frac{\partial f}{\partial \bar{z}_{j}}(z)\right\rangle_{\mathcal{H}} \\
& =B\left(\frac{\partial f}{\partial \bar{z}_{j}}(z),(\psi \otimes \bar{h})(z)\right),
\end{aligned}
$$

hence $\left\langle\left(\frac{\partial f}{\partial \bar{z}_{j}}\right)_{h}, \psi\right\rangle$ also equals $\left\langle\frac{\partial f}{\partial \bar{z}_{j}},(\psi \otimes \bar{h})\right\rangle$. This proves the first assertion of the lemma. In the particular case $\bar{\partial} f=0$, one gets immediately $\bar{\partial} f_{h}=0$ in the usual distribution sense. The second assertion follows.

Now let $\left(e_{\nu}\right)_{\nu \in \mathbb{Z}}$ be a Hilbert basis for $\mathcal{H}$. For any map $f: \Omega \longrightarrow$ $\Lambda^{(0, q)} \otimes \mathcal{H}, f=\sum_{Q} d \bar{z}^{Q} \otimes f_{Q}$, we define the $\nu^{\text {th }}$ component $f_{\nu}$ of $f$ by

$$
f_{\nu}(z)=\sum_{Q}\left\langle e_{\nu} \mid f_{Q}(z)\right\rangle_{\mathcal{H}} d \bar{z}^{Q}, \quad z \in \Omega
$$


In particular, for $q=0$, one has $f_{\nu}(z)=\left\langle e_{\nu} \mid f(z)\right\rangle_{\mathcal{H}}=f_{e_{\nu}}(z)$ in the sense of (1.1.1). The following properties are then easy to check:

(i) For any $z$ in $\Omega$, the equality $f(z)=\sum_{\nu \in \mathbb{Z}} f_{\nu}(z) \otimes e_{\nu}$ holds in $\Lambda^{(0, q)} \otimes \mathcal{H}$, and consequently $\|f(z)\|_{\mathcal{H}}^{2}=\sum_{\nu \in \mathbb{Z}}\left|f_{\nu}(z)\right|^{2}$.

(ii) If $f$ belongs to $L_{(0, q)}^{2}(\Omega ; \mathcal{H}$, loc $)$, then each $f_{\nu}$ belongs to $L_{(0, q)}^{2}(\Omega ; \mathbb{C}$, loc $)$ and the equality $f=\sum_{\nu \in \mathbb{Z}} f_{\nu} \otimes e_{\nu}$ holds in

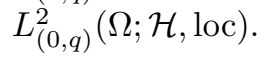

(iii) If moreover $\bar{\partial} f$ belongs to $L_{(0, q+1)}^{2}(\Omega ; \mathcal{H}$, loc $)$, then for each $\nu$ the form $\bar{\partial}\left(f_{\nu}\right)$ belongs to $L_{(0, q+1)}^{2}(\Omega ; \mathbb{C}$, loc $)$ and satisfies $\bar{\partial}\left(f_{\nu}\right)=$ $(\bar{\partial} f)_{\nu}$.

Although simple, these properties are the key to the following Hörmander-type result.

1.4. Proposition. Let $\varphi$ be a continuous plurisubharmonic function in $\Omega$. Let $q$ be an integer, $q \geq 0$. Then for any form $f$ in $L_{(0, q+1)}^{2}(\Omega ; \mathcal{H}$, loc $)$ satisfying $\bar{\partial} f=0$ and

$$
\int_{\Omega}\|f(z)\|_{\mathcal{H}}^{2} e^{-\varphi(z)} d V(z)<\infty
$$

there exists a form $g$ in $L_{(0, q)}^{2}(\Omega ; \mathcal{H}$, loc $)$ such that $\bar{\partial} g=f$ and

$$
\int_{\Omega}\|g(z)\|_{\mathcal{H}}^{2} e^{-\varphi(z)} d V(z) \leq C_{\Omega} \int_{\Omega}\|f(z)\|_{\mathcal{H}}^{2} e^{-\varphi(z)} d V(z),
$$

where $C_{\Omega}$ is a positive constant depending only on the diameter of $\Omega$.

Proof: By properties (i)-(iii) above, it is easily seen that for each $\nu$, the form $f_{\nu}$ lies in $L_{(0, q+1)}^{2}(\Omega ; \mathbb{C}$, loc $)$, is $\bar{\partial}$-closed, and has finite weighted norm $\int_{\Omega}\left|f_{\nu}(z)\right|^{2} e^{-\varphi(z)} d V(z)$. Applying a standard result of Hörmander $([\mathbf{5}, 4.4 .2])$, we solve $\bar{\partial}$ componentwise: one gets a form $g_{\nu}$ in $L_{(0, q)}^{2}(\Omega ; \mathbb{C}$, loc $)$ such that $\bar{\partial} g_{\nu}=f_{\nu}$ and $\int_{\Omega}\left|g_{\nu}(z)\right|^{2} e^{-\varphi(z)} d V(z) \leq$ $C_{\Omega} \int_{\Omega}\left|f_{\nu}(z)\right|^{2} e^{-\varphi(z)} d V(z)$. This, together with the elementary fact $\left\|g_{\nu}(z) \otimes e_{\nu}\right\|_{\mathcal{H}}=\left|g_{\nu}(z)\right|$, allows easily to check that the series $\sum_{\nu \in \mathbb{Z}} g_{\nu} \otimes e_{\nu}$ converges in $L_{(0, q)}^{2}(\Omega ; \mathcal{H}$, loc $)$ to a form $g$ satisfying all the required properties. 
1.5. Remark. When the Hilbert space $\mathcal{H}$ is chosen as a Sobolev space (this choice will be adopted in the next section), Proposition 1.4 can be viewed as a result on parameter dependence for $\bar{\partial}$ in weighted $L^{2}$ spaces, with a weight which does not depend on the parameter. Different results for parameter-depending weights can be found in [4].

\section{Construction of a barrier map}

2.1. Setting of the construction. Let $U$ and $k$ be as in the introduction. From now on, the Hilbert space $\mathcal{H}$ will be the Sobolev space $H^{m}(U)$ with $m=n+k+1$. By Sobolev's lemma, this choice of $m$ ensures the inclusion $H^{m}(U) \subset C^{k}(\bar{U})$ with continuous injection (this is the only reason why some regularity for $\partial \Omega$-namely $\mathrm{Lip}^{1}$, see e.g. $[\mathbf{1}]-$ is required in this work). There exists a positive constant $A_{k}$ depending only on $k, \Omega$ and $\Omega^{*}$, such that for any integer $\ell$ with $0 \leq \ell \leq k$ and any derivation $D_{\zeta}^{\ell}$ of order $\ell$, the estimate

$$
\left|D_{\zeta}^{\ell} F(\zeta)\right| \leq A_{k}\|F\|_{H^{m}(U)}
$$

holds for any $\zeta \in \bar{U}$ and any $F \in H^{m}(U)$. In particular, $F \mapsto F(\zeta)$ is a continuous linear form on the Hilbert space $H^{m}(U)$, so there exists $h(\zeta)$ in $H^{m}(U)$ such that one has $F(\zeta)=\langle h(\zeta) \mid F\rangle_{H^{m}(U)}$ for any $F \in H^{m}(U)$. Consequently, any map $f$ belonging to $L^{2}\left(\Omega ; H^{m}(U)\right.$, loc $)$ satisfies, with the notation (1.1.1),

$$
f(z, \zeta)=f_{h(\zeta)}(z) \text { for all }(z, \zeta) \in \Omega \times \bar{U} .
$$

This, together with Lemma 1.3, implies clearly the following fact: if $\bar{\partial} f$ (in the sense of section 1) belongs to $L_{(0,1)}^{2}\left(\Omega ; H^{m}(U)\right.$, loc), then for any $(z, \zeta) \in \Omega \times \bar{U}$, one can view $(\bar{\partial} f)(z, \zeta)$ as $\bar{\partial}_{z}(f(z, \zeta))$ computed in the usual space $L_{(0,1)}^{2}(\Omega ; \mathbb{C}$, loc $)$, with $\zeta$ fixed. This remark extends obviously to forms of higher degrees.

Now we follow the pattern of the classical work of L. Hörmander [6]. For any integer $s$ and any real $\gamma$ with $s \geq 0, \gamma \geq 0$, let $L_{\gamma}^{s, q}$ be the space of families $w=\left(w_{I} ; I \in\{1, \ldots, n\}^{s}\right)$ of elements of $L_{(0, q)}^{2}\left(\Omega ; H^{m}(U)\right.$, loc $)$ satisfying, for each multi-index $I \in\{1, \ldots, n\}^{s}$, both following properties:

$$
\int_{\Omega}\left\|w_{I}(z, \cdot)\right\|_{H^{m}(U)}^{2} \operatorname{dist}(z, \partial \Omega)^{2 \gamma} d V(z)<\infty
$$

(2.1.4) the map

$$
\left(t^{1}, \ldots, t^{s}\right) \longmapsto \sum_{I \in\{1, \ldots, n\}^{s}} t_{i_{1}}^{1} \ldots t_{i_{s}}^{s} w_{I}
$$

is $s$-linear alternating with respect to $\left(t^{1}, \ldots, t^{s}\right) \in\left(\mathbb{C}^{n}\right)^{s}$. 
The $\bar{\partial}$-operator defined in section 1 extends to $L_{\gamma}^{s, q}$ by putting $\bar{\partial} w=$ $\left(\bar{\partial} w_{I} ; I \in\{1, \ldots, n\}^{s}\right)$.

For $j=1, \ldots, n$ and for any $f$ in $L^{2}\left(\Omega ; H^{m}(U)\right.$, loc $)$, let $\sigma_{j} f$ be the map which, to each point $z \in \Omega$, associates the function

$$
\left(\sigma_{j} f\right)(z, \cdot): \zeta \in U \longmapsto\left(z_{j}-\zeta_{j}\right) f(z, \zeta) .
$$

It is not difficult to see that $\sigma_{j} f$ itself belongs to $L^{2}\left(\Omega ; H^{m}(U)\right.$, loc $)$ and that for any $z \in \Omega$, one has

$$
\left\|\left(\sigma_{j} f\right)(z, \cdot)\right\|_{H^{m}(U)} \leq C\|f(z, \cdot)\|_{H^{m}(U)}
$$

with a suitable positive constant $C$, depending only on $m$ and on the size of $\Omega, \Omega^{*}$. In particular, $\sigma_{j}$ acts componentwise as a continuous linear operator on all the spaces $L_{(0, q)}^{2}\left(\Omega ; H^{m}(U)\right.$, loc $)$.

Now let $w$ be an element of $L_{\gamma}^{s+1, q}$. For each $I=\left(i_{1}, \ldots, i_{s}\right)$ in $\{1, \ldots, n\}^{s}$, we put

$$
(P w)_{I}=\sum_{j=1}^{n} \sigma_{j} w_{(I, j)}
$$

with $(I, j)=\left(i_{1}, \ldots, i_{s}, j\right)$. Note that for $(z, \zeta) \in \Omega \times U$, one gets explicitely

$$
(P w)_{I}(z, \zeta)=\sum_{j=1}^{n}\left(z_{j}-\zeta_{j}\right) w_{(I, j)}(z, \zeta)
$$

Also, by the properties of $\sigma_{j}$ described above (especially (2.1.5)), this defines an element $P w$ of $L_{\gamma}^{s, q}$. It is straightforward to check that the operator $P$ commutes with $\bar{\partial}$ and that it satisfies $P^{2}=0$, giving a double complex just as in $[\mathbf{6}]$.

2.2. Lemma. Let $w$ be an element of $L_{\gamma}^{s, q+1}$ such that $\bar{\partial} w=0$. Then there exists $v$ in $L_{\gamma}^{s, q}$ such that $\bar{\partial} v=w$.

Proof: Obvious by Proposition 1.4 applied with

$$
\varphi(z)=-2 \gamma \log \operatorname{dist}(z, \partial \Omega)
$$


2.3. Lemma. Let $v$ be an element of $L_{\gamma}^{s, q}$ such that $P v=0$. Then there exists $w$ in $L_{\gamma+m+1}^{s+1, q}$ such that $P w=v$. If one has additionally $\bar{\partial} v=0$, then $\bar{\partial} w$ belongs to $L_{\gamma+m+2}^{s+1, q+1}$.

Proof: Following [6, Lemma 6], we define explicitely

$$
w_{I}(z, \zeta)=\sum_{k=1}^{s+1}(-1)^{s+1-k} \frac{\bar{z}_{i_{k}}-\bar{\zeta}_{i_{k}}}{|z-\zeta|^{2}} v_{I \backslash\left(i_{k}\right)}(z, \zeta)
$$

for $I=\left(i_{1}, \ldots, i_{s+1}\right) \in\{1, \ldots, n\}^{s+1},(z, \zeta) \in \Omega \times U$ and $I \backslash\left(i_{k}\right)=" I$ with the index $i_{k}$ deleted". Just as in [6], one gets $P w=v$ and part (2.1.4) of the definition of $L_{\gamma}^{s+1, q}$ is satisfied. The only things to check are the growth conditions. But for any $(z, \zeta) \in \Omega \times U$ and any derivation $D_{\zeta}^{\ell}$ of order at most $m$, it is clear that

$$
\left|D_{\zeta}^{\ell}\left(\frac{\bar{z}_{i_{k}}-\bar{\zeta}_{i_{k}}}{|z-\zeta|^{2}}\right)\right| \leq C^{\prime} \operatorname{dist}(z, \partial \Omega)^{-(m+1)}
$$

hence

$$
\left\|w_{I}(z, \cdot)\right\|_{H^{m}(U)}^{2} \leq C^{\prime \prime} \operatorname{dist}(z, \partial \Omega)^{-2(m+1)} \sum_{k=1}^{s+1}\left\|v_{I \backslash\left(i_{k}\right)}(z, \cdot)\right\|_{H^{m}(U)}^{2}
$$

for suitable positive constants $C^{\prime}$ and $C^{\prime \prime}$, depending only on $m$ and on the size of $\Omega, \Omega^{*}$. From these estimates, it is not difficult to prove that $w$ belongs to $L_{\gamma+m+1}^{s+1, q}$. The claim that $\bar{\partial} w$ belongs to $L_{\gamma+m+2}^{s+1, q+1}$ when $\bar{\partial} v$ vanishes comes similarly, since one gets then

$$
\left(\bar{\partial} w_{I}\right)(z, \zeta)=\sum_{k=1}^{s+1}(-1)^{s+1-k} \bar{\partial}_{z}\left(\frac{\bar{z}_{i_{k}}-\bar{\zeta}_{i_{k}}}{|z-\zeta|^{2}}\right) \wedge v_{I \backslash\left(i_{k}\right)}(z, \zeta),
$$

by elementary computations using the remarks in 2.1 .

We are now ready to state the key result of this paper.

2.4. Theorem. For every element $v$ of $L_{\gamma}^{s, q}$ satisfying $\bar{\partial} v=0$ and $P v=0$, there exists an element $w$ of $L_{\gamma+m+1+\left(\frac{m}{2}+1\right)(2 n-q-s)}^{s+1, q}$ such that $\bar{\partial} w=0$ and $P w=v$. 
Proof: We essentially mimic the proof of Theorem 7 in [6], by decreasing induction on $q$ and $s$. The only different point is that a special attention has to be paid to the evolution of the index $\gamma$ (invisible in [6]) during the induction. First, the result is trivial for $q>n$ or $s>n$. Now assume that it holds for data in $L_{\gamma^{\prime}}^{s+1, q+1}$ where $\gamma^{\prime}$ is an arbitrary positive real number, and $s \leq n, q \leq n$. Let $v$ be as in the statement of the theorem. By 2.3, there exists $w^{\prime}$ in $L_{\gamma+m+1}^{s+1, q}$ such that $P w^{\prime}=v$ and $\bar{\partial} w^{\prime} \in L_{\gamma+m+2}^{s+1, q+1}$. Since $\bar{\partial}\left(\bar{\partial} w^{\prime}\right)=0$ and $P\left(\bar{\partial} w^{\prime}\right)=\bar{\partial}\left(P w^{\prime}\right)=\bar{\partial} v=0$, the induction assumption applies to the data $\bar{\partial} w^{\prime}$ with $\gamma^{\prime}=\gamma+m+2$. One gets $w^{\prime \prime}$ in $L_{\gamma^{\prime \prime}}^{s+2, q+1}$ with $\gamma^{\prime \prime}=\gamma^{\prime}+m+1+\left(\frac{m}{2}+1\right)(2 n-(q+1)-(s+1))=$ $\gamma+m+1+\left(\frac{m}{2}+1\right)(2 n-q-s)$, such that $P w^{\prime \prime}=\bar{\partial} w^{\prime}$ and $\bar{\partial} w^{\prime \prime}=0$. By 2.2 , we can find $w^{\prime \prime \prime}$ in $L_{\gamma^{\prime \prime}}^{s+2, q}$ such that $\bar{\partial} w^{\prime \prime \prime}=w^{\prime \prime}$. Put $w=w^{\prime}-P w^{\prime \prime \prime}$. Since one has $w^{\prime} \in L_{\gamma+m+1}^{s+1, q} \subset L_{\gamma^{\prime \prime}}^{s+1, q}$, we see that $w$ belongs to $L_{\gamma^{\prime \prime}}^{s+1, q}$. Finally one checks that $\bar{\partial} w=\bar{\partial} w^{\prime}-\bar{\partial}\left(P w^{\prime \prime \prime}\right)=P w^{\prime \prime}-P\left(\bar{\partial} w^{\prime \prime \prime}\right)=0$ and $P w=P w^{\prime}=v$.

2.5. Proof of the main theorem of the introduction: The application of 2.4 with $q=s=\gamma=0$ and $v \equiv 1$ yelds functions $w_{1}, \ldots, w_{n}$ belonging to $L^{2}\left(\Omega ; H^{m}(U)\right.$, loc $)$ and satisfying the following properties:

$$
\begin{gathered}
\left.\bar{\partial} w_{j}=0 \text { (in the sense of section } 1\right) \text { for } j=1, \ldots, n, \\
\sum_{j=1}^{n} w_{j}(z, \zeta)\left(z_{j}-\zeta_{j}\right)=1 \text { for any }(z, \zeta) \in \Omega \times U, \\
\int_{\Omega}\left\|w_{j}(z, \cdot)\right\|_{H^{m}(U)}^{2} \operatorname{dist}(z, \partial \Omega)^{2(n+1)(m+2)-2} d V(z)<\infty \\
\text { for } j=1, \ldots, n
\end{gathered}
$$

Observe that property (2.5.2) is true as well for $\zeta \in \bar{U}$, since the functions $w_{j}(z, \cdot)$ extend continuously to $\bar{U}$ for all fixed $z \in \Omega$ (see 2.1). Also, in virtue of Lemma 1.3, of the representation formula (2.1.2) and of (2.5.1), the function $z \mapsto w_{j}(z, \zeta)$ is holomorphic in $\Omega$ for each $\zeta \in \bar{U}$. By the same ideas, this can be shown as well for the functions $D_{\zeta}^{\ell} w_{j}(\cdot, \zeta)$, $\ell \leq k$. Thus we have proved part (1) and (2) of the main theorem. We proceed now to prove part (3). For $z \in \Omega$, let $B_{z}$ be the euclidean ball with center $z$ and radius $\frac{1}{2} \operatorname{dist}(z, \partial \Omega)$. The plurisubharmonicity of $\left|D_{\zeta}^{\ell} w_{j}(\cdot, \zeta)\right|^{2}$ yelds

$$
\left|D_{\zeta}^{\ell} w_{j}(z, \zeta)\right|^{2} \leq C \operatorname{dist}(z, \partial \Omega)^{-2 n} \int_{B_{z}}\left|D_{\zeta}^{\ell} w_{j}(t, \zeta)\right|^{2} d V(t)
$$


for some constant $C$. Besides, one has $1 \leq 2 \operatorname{dist}(z, \partial \Omega)^{-1} \operatorname{dist}(t, \partial \Omega)$ for all $t \in B_{z}$. From this, and from the estimate (2.1.1) applied to $F=w_{j}(t, \cdot)$, we deduce

$$
\begin{aligned}
\left|D_{\zeta}^{\ell} w_{j}(z, \zeta)\right|^{2} \leq & C^{\prime} \operatorname{dist}(z, \partial \Omega)^{-2(n+(m+2)(n+1))+2} \\
& \times \int_{\Omega}\left\|w_{j}(t, \cdot)\right\|_{H^{m}(U)}^{2} \operatorname{dist}(t, \partial \Omega)^{2(n+1)(m+2)-2} d V(t),
\end{aligned}
$$

for some suitable constant $C^{\prime}$ depending on $k, \Omega, \Omega^{*}$. Taking into account (2.5.3) and the value $m=n+k+1$, this proves part (3) of the main theorem. Now we end by showing how to obtain $C^{k}$ regularity with respect to both variables $(z, \zeta) \in \Omega \times \bar{U}$. This requires actually standard arguments, so we will just sketch the proof for $C^{0}$ regularity. Pick $\left(z^{0}, \zeta^{0}\right) \in \Omega \times \bar{U}$ and put $E_{1}=w_{j}(z, \zeta)-w_{j}\left(z^{0}, \zeta\right), E_{2}=w_{j}\left(z^{0}, \zeta\right)-w_{j}\left(z^{0}, \zeta^{0}\right)$. One has clearly $\left|E_{1}\right| \leq C\left(z^{0}, \zeta\right)\left|z-z^{0}\right|$ with

$$
C\left(z^{0}, \zeta\right)=\operatorname{Sup}_{1 \leq p \leq n, t \in B_{z^{0}}}\left|\frac{\partial}{\partial t_{p}} w_{j}(t, \zeta)\right| .
$$

But in virtue of the holomorphy of $w_{j}(t, \zeta)$ with respect to $t$, of the Cauchy formula and of the estimate (3) of the theorem, we see that $C\left(z^{0}, \zeta\right)$ is bounded uniformly with respect to $\zeta \in \bar{U}$. Thus $E_{1}$ tends to 0 uniformly with respect to $\zeta$ when $z$ tends to $z^{0}$. On the other hand, since $w_{j}\left(z^{0}, \cdot\right)$ is continuous, it is clear that $E_{2}$ tends to 0 when $\zeta$ tends to $\zeta^{0}$. As announced, one gets finally that $w_{j}(z, \zeta)$ tends to $w_{j}\left(z^{0}, \zeta^{0}\right)$ as $(z, \zeta)$ tends to $\left(z^{0}, \zeta^{0}\right)$.

2.6. Concluding remark. The results described above suggest a question: is it possible to obtain valuable information about parameter dependence in Skoda's theorem ([13, Théorème 1]) by the kind of argument used in the present paper ? It is known (see e.g. [3], [7], [12]) that such a result would have significant consequences in the construction of integral operators solving the $\bar{\partial}$-equation.

\section{References}

1. R. Adams, "Sobolev spaces," Academic Press, 1975.

2. N. Bourbaki, "Intégration 1-4," Hermann, 1965.

3. J. Chaumat and A.-M. Chollet, Noyaux pour résoudre l'équation $\bar{\partial}$ dans des classes ultradifférentiables sur des compacts irréguliers de $\mathbb{C}^{n}$, in "Proceedings of the special year on complex analysis," 
Mittag-Leffler Inst. 1987-1988, Math. Notes 38, Princeton University Press, 1993, pp. 205-226.

4. K. Diederich AND T. OHsawa, On the parameter dependence of solutions to the $\bar{\partial}$-equation, Math. Ann. 289 (1991), 581-587.

5. L. HÖRMANDER, "An introduction to complex analysis in several variables," 3rd edition, North-Holland, 1994.

6. L. HÖRMANDER, Generators for some rings of analytic functions, Bull. Amer. Math. Soc. 73 (1967), 943-949.

7. J. MiCHEL, Integral representations on weakly pseudoconvex domains, Math. Z. 208 (1991), 437-462.

8. J. MiChel AND M.-C. SHAW, A decomposition problem on weakly pseudoconvex domains, Math. Z. (to appear).

9. J. MiChel AND M.-C. SHAW, $C^{\infty}$-regularity of solutions of the tangential CR equations on weakly pseudoconvex manifolds, Math. Ann. (to appear).

10. J. Michel AND M.-C. Shaw, The $\bar{\partial}$ problem on domains with piecewise smooth boundaries with applications, Preprint (September 1997).

11. J. Michel AND M.-C. Shaw, $\bar{\partial}$ and $\bar{\partial}_{b}$ problems on non-smooth domains, Preprint (January 1998).

12. R. M. RANGE, Integral kernels and Hölder estimates for $\bar{\partial}$ on pseudoconvex domains of finite type in $\mathbb{C}^{2}$, Math. Ann. 288 (1990), 63-74.

13. H. SkodA, Applications des techniques $L^{2}$ à la théorie des idéaux d'une algèbre de fonctions holomorphes avec poids, Ann. Sci. École Norm. Sup. 5 (1972), 545-579.

\author{
CNRS - URA 751 \\ Mathématiques - Bâtiment M2 \\ Université des Sciences et Technologies de Lille \\ 59655 Villeneuve d'Ascq Cedex \\ FRANCE \\ e-mail: Vincent.Thilliez@univ-lille1.fr
}

Primera versió rebuda el 10 de setembre de 1997 , darrera versió rebuda el 16 de febrer de 1998 